\title{
Anomalous Conductance Quantization in the Inter-band Gap of a One-dimensional Channel
}

\author{
Frederick Green ${ }^{1}$ and Mukunda P. Das ${ }^{2}$ \\ ${ }^{1}$ School of Physics, The University of New South Wales, Sydney, NSW 2052, Australia. \\ ${ }^{2}$ Department of Theoretical Physics, RSPE, The Australian National University, Canberra, \\ ACT 2601, Australia.
}

\begin{abstract}
We report on a striking departure from the canonical step sequence of quantized conductance in a ballistic, quasi-one-dimensional metallic channel. Ideally, in such a structure, each sub-band population contributes its Landauer conductance quantum independently of the rest. In a picture based exclusively on coherent single-carrier transmission, unitary backscattering can lower a conductance step below ideal, but it is absolutely impossible for it to enhance the ideal Landauer conductance of a sub-band. Precisely such an anomalous and robust nonlinear enhancement has already been observed over the whole density range between sub-band thresholds (de Picciotto R et al 2004 Phys. Rev. Lett. 92036805 and 2008 J. Phys. Condens. Matter 20 164204). We show theoretically that the anomalous enhancement of ideal Landauer conductance is the hallmark of carrier transitions coupling the discrete sub-bands.
\end{abstract}

\section{Introduction}

Understanding the conductance in quantum-confined metallic channels is a central aspect of electrical transport in meso- and nanoscopic structures. Quantization of the conductance in ballistic quantum-well channels is the unique property of their one-dimensional (1D), waveguidelike nature.

The standard model of 1D quantized conductance [1, 2, 3] adopts the viewpoint of Landauer and associates [4, 5] in which a highly constricted channel, interposed between macroscopic ohmic contacts, is conceived as a simple barrier potential modifying the propagation of free singleelectron quantum states. Strong lateral confinement of these states by the device's quantum-well structure leads to their segregation into discrete levels (sub-bands), separated by energy gaps whose magnitude may run from tens of $\mathrm{meV}$ in III-V heterojunctions to several $\mathrm{eV}$ in carbon nanotubes.

This picture of conductance as quantum transmission accounts elegantly for the welldocumented resolution of the ohmic conductance in 1D structures into a sequence of integral plateaux. As a function of increasing carrier density in the device, each successive step in the conductance extends, unaltered, throughout the energy-gap region separating the discrete subbands. A new plateau appears as soon as the chemical potential and thus the carrier population cross the gap to access the next higher sub-band.

That the single-particle quantum transmission picture does not address all experimental observations is known [6, 7, [8, 9]. The reasons that it does not are also known [10]. First, quantum transmission theory is restricted to weak-field linear response; second, it does not account for the resistive dissipation that is inevitable in every ohmic structure; third, the 
approach has no way to address scattering processes other than purely coherent, purely elastic, back-scattering. These and other fundamental drawbacks of the transmission approach have been critiqued in detail elsewhere [11, 12, 13.

One example of a crucial inelastic physical process is intra-band scattering by phonon emission, responsible for the above-mentioned dissipation. Another example is inter-band transitions, which are inherently inelastic two-body processes with substantial energy exchange between discrete bands. They lie beyond the scope of a single-carrier description.

The physics of discrete transitions between distinct conduction sub-bands lies at the heart of this paper. The experimental findings that animate our work are those of de Picciotto, Pfeiffer, Baldwin and West [8, 9.

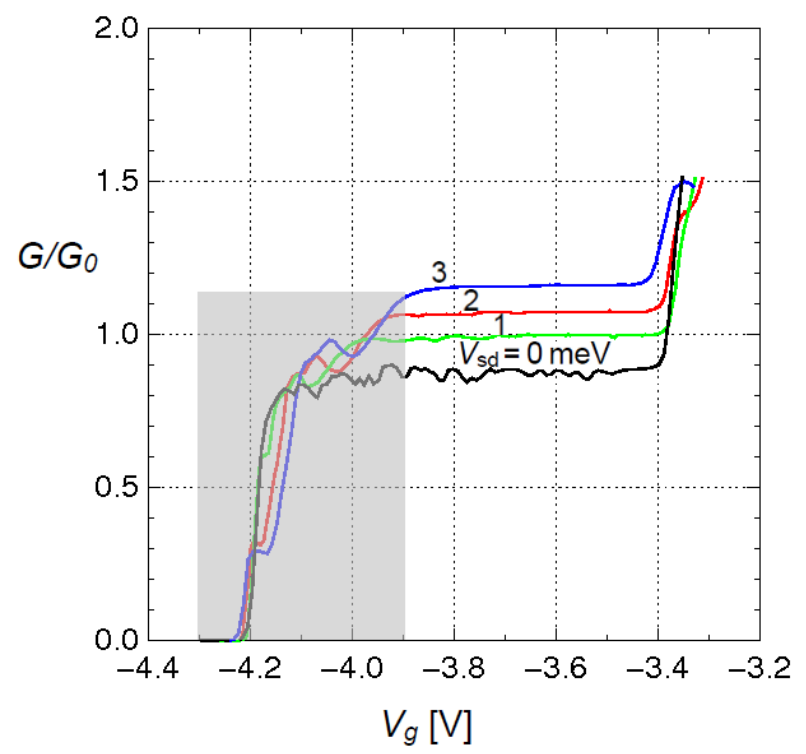

FIG. 1 Quantized differential conductance measured in a quasi-one-dimensional multi-sub-band ballistic channel, after de Picciotto et al, reference 9. Conductance is plotted in Landauer units $G_{0}=77.48 \mu \mathrm{S}$, as a function of a control-gate voltage modulating the total carrier density within the channel. Beyond the labile structures (grey box) at threshold of the ground-state band lies a series of very flat extended plateaux observed at different values of source-drain bias voltage inducing the current. The system is taken beyond linear response, as seen in the bias dependence of the conductance. Most remarkable is the raising of $G$ above the absolute upper limit $G_{0}$ imposed by unitarity. This shows that effects beyond simple quantum-coherent transmission dominate the transport physics.

Let us explain the importance of the remarkable non-linear, non-Landauer 1D conductance plateaux documented by de Picciotto and colleagues. Figure 1 reproduces the core results of their references [8] and [9]. It shows a series of differential-conductance traces (normalized to the Landauer quantum $G_{0}=77.48 \mu \mathrm{S}$ ) for a nearly ideal ballistic quantum wire, taken at fixed source-drain driving voltage and plotted as functions of gate voltage controlling the channel chemical potential and so its carrier density. The greyed region contains a complex of highly mutable shoulder structures evident at the ground-state threshold, popularly termed the " 0.7 anomaly", which is not of interest here.

We focus, by contrast, upon the conductance plateaux extending over the larger part of the inter-band region up to the threshold of the first excited sub-band. Unlike the " 0.7 " features [14, they are robustly regular with a highly systematic dependence on the source-drain driving voltage.

- The anomalous steps are extremely flat and extend, with carrier density, from the early- 
onset "0.7" feature sequence right up to the threshold of the next higher sub-band. Qualitatively and quantitatively, they are wholly distinct from the relatively ephemeral structures close to first threshold.

- The anomalous conductance steps are voltage dependent. They are beyond any linearresponse description.

- These plateaux cover the entire region where the chemical potential of the carriers lies in the gap between ground- and first-excited-state bands. According to quantum-transmission theory, such a structure cannot be higher than the ideal limit $G_{0}$.

- With increasing source-drain voltage, the enhanced steps increase in size from the expected weak-field baseline, exceeding appreciably the absolute maximum set by $G_{0}$. We stress the impossibility of such a scenario within quantum transmission, which would otherwise see its unitarity (conservation of probability) wrecked.

The devices studied in references [8] and [9] are of unprecedented quality, perhaps the closestto-perfect ballistic wires so far fabricated. We remark on the care taken by the cited authors to isolate the physics at work within their samples. It is a little surprising, then, that (to our knowledge) no authors since the original team [9] have commented on the startling violation of accepted predictions by the quantized conductance data of figure 1 .

Reference [8] contains a prescient comment on the role of inter-mode coupling within the test structures; that is, that there should be some exchange of energy and possibly carriers between sub-bands, setting up a mutual dynamical feedback. To date, theoretical support for that hypothesis has not been at hand. The goal is to provide it.

In the following we present a brief description of our quantum kinetic analysis of the anomalous steps in $1 \mathrm{D}$ conductance. Our microscopically conserving model is based on the quantum Boltzmann formalism [10] extended to inter-band transitions. After this short account we survey our numerical results, comparing and contrasting them with the basic data of de Picciotto and colleagues. Particular features of the results shed light on the relevant physics. The paper ends with a summary and foreshadows novel theoretical possibilities that could be tested in a renewed series of experiments in similar high-quality structures.

\section{Problem and Solution}

The problem is to try to replicate and thus unpack the physics of anomalous non-linear enhancement of the Landauer conductance. We posit a uniform one-dimensional ballistic channel.

Since, even in principle, the active 1D device region cannot be divorced from the large source and drain boundary leads, its operative length is no longer exclusively determined by its physical dimensions or the bulk mean free paths of its originating substrate. Rather, its length is dictated by the longest carrier mean free path (MFP) for the channel as embedded in its non-ideal bounding leads. Adopting the estimate suggested in reference [8, we take an operational channel length $L=2 \mu \mathrm{m}$. Our uniform-channel results, however, do not depend on the absolute MFPs assumed for this ballistic scenario.

In essence we are describing carrier behaviour averaged over an abstract ensemble of such wires, seamlessly connected in series, each with maximum mean free path $L$. At cryogenic temperatures one expects the inelastic mean free path to set the longer scale, fixing $L$. On the other hand, the observed weak-field conductance falls short of the ideal Landauer quantum and we set the complementary elastic intra-band MFP somewhat below $L$. An elastic MFP of $0.769 \mathrm{~L}$ matches the mean weak-field conductance after reference [8]. 


\subsection{Transport equations with inter-band coupling}

Our transport equations define the behaviour of two sub-band populations, separated by their energy gap $E_{g}$. Given two steady-state distributions $f_{k j}$ for the lower band $(j=1)$ and the next higher $(j=2)$ as functions of band momentum $k$, the equations are of modified BoltzmannDrude form:

$$
\begin{array}{cc}
\frac{q \mathcal{E}}{\hbar} \frac{\partial f_{k 1}}{\partial k}=\quad- & R_{\mathrm{in} 1}\left(f_{k 1}-\bar{f}_{k 1}\right)-R_{\mathrm{e} 11} f_{k 1}^{o} \\
& -R_{01}\left(e^{-E_{g} / k_{\mathrm{B}} T} f_{k 1}\left(1-\bar{f}_{k 2}\right)-\bar{f}_{k 2}\left(1-f_{k 1}\right)\right), \\
\frac{q \mathcal{E}}{\hbar} \frac{\partial f_{k 2}}{\partial k}=\quad-R_{\mathrm{in} 2}\left(f_{k 2}-\bar{f}_{k 2}\right)-R_{\mathrm{el} 2} f_{k 2}^{o} \\
\quad-R_{02}\left(f_{k 2}\left(1-\bar{f}_{k 1}\right)-e^{-E_{g} / k_{\mathrm{B}} T} \bar{f}_{k 1}\left(1-f_{k 2}\right)\right) .
\end{array}
$$

The nature of the reference distributions $\bar{f}_{k j}$ is explained below. Other notation is as follows. On the left-hand side of this pair of steady-state equations, $\mathcal{E}$ is the uniform field exerted on the carriers by the applied source-drain voltage. On the right-hand sides the parameters $R_{\mathrm{in} j}$ and $R_{\mathrm{el} j}$ are, respectively, the intra-band inelastic and elastic scattering rates assigned to each band while $R_{0 j}$ correspondingly is the inter-band transition rate for the coupling between the two populations. The elastic collision term scales with the odd part of the distributions since elastic scattering can only reverse the momentum direction with no change in energy: $f_{k j}^{o}=\left(f_{k j}-f_{-k j}\right) / 2$.

The final parameter is the Boltzmann factor $e^{-E_{g} / k_{\mathrm{B}} T}$ associated with promoting a carrier from lower to upper sub-band across their gap separation $E_{g}$. In the subsequent discussion we will express all energies in thermal units $k_{\mathrm{B}} T$, and momenta in thermal units $k_{\mathrm{th}} \equiv \sqrt{2 k_{\mathrm{B}} T / \hbar^{2}}$.

We now discuss the meaning and role of the effective equilibrium functions

$$
\begin{aligned}
\bar{f}_{k 1}(\mu) & \equiv 1 /\left(1+\exp \left(k^{2}-\mu\right)\right) \text { and } \\
\bar{f}_{k 2}\left(\mu-E_{g}\right) & \equiv 1 /\left(1+\exp \left(k^{2}+E_{g}-\mu\right)\right)
\end{aligned}
$$

with momenta and energies in thermal units. Transition events redistribute the electron population between the two sub-bands of the channel. The change in their respective densities is presumed to depress the effective chemical potential of the lower band: $\mu$ is renormalized to $\mu-\zeta_{1}$ while the augmented population in the upper band, located above the lower by the band gap $E_{g}$, follows the rise in its effective chemical potential: $\mu-E_{g}$ goes to $\mu+\zeta_{2}-E_{g}$. Thus both quantities $\zeta_{1}$ and $\zeta_{2}$ should be non-negative.

For any choice of the pair of renormalized chemical potentials, the coupled equations (1) are solved systematically with the expressions for $\bar{f}_{k 1}\left(\mu-\zeta_{1}\right)$ and $\bar{f}_{k 2}\left(\mu+\zeta_{2}-E_{g}\right)$, equations (2), as input. Details of the relevant Green-function algorithm are left to a longer account.

\subsection{Microscopic conservation}

Consider the equilibrium state at zero field, for which $\zeta_{1}=0=\zeta_{2}$. The effective equilibria are now absolute and furthermore $f_{k j}=\bar{f}_{k j}$. The distinct terms on either side of the transport equations (1) all vanish individually. Detailed balance is satisfied.

What happens at finite field? The intra- and inter-band inelastic collision terms on the righthand sides of equations (1) are not guaranteed to vanish identically when integrated separately, although the left-hand-side expressions always do so. As a consequence conservation must be imposed explicitly upon the solution to the joint response. [11, 15, 16] We have a type of 
generation-recombination problem; it follows that conservation cannot apply to each sub-band individually, but only jointly.

The bi-linear coupling between distributions in the inter-band transition terms means that the full problem cannot be solved even semi-analytically save in the trivial case of independent sub-bands (zero transitions, $R_{01}=R_{02}=0$ ). Conservation then appears as a mandatory relation linking the pair of potential shifts $\zeta_{j}$ so the system's total density is invariant (the spin factor appears explicitly in the integrals, which are rendered in dimensionless units):

$$
\begin{aligned}
& 2 \int d k\left(\bar{f}_{k 1}(\mu)+\bar{f}_{k 2}\left(\mu-E_{g}\right)\right) \equiv n(\mu) \\
& \quad \equiv 2 \int d k\left(f_{k 1}\left(\mu-\zeta_{1} ; \zeta_{2}\right)+f_{k 2}\left(\mu+\zeta_{2}-E_{g} ; \zeta_{1}\right)\right)
\end{aligned}
$$

Since there are two undetermined quantities to solve, a second relation is needed. The new physical information to be adduced must be independent of anything contained in the transport equations themselves.

For the second, constitutive relation we take the Helmholtz free-energy density for the nonequilibrium carrier distribution and remove from it the formal energy of assembly for the system, mediated by the chemical potential. Under the action of the external driving field, the change in this net energy measures the internal dynamical rearrangement of the sub-band distributions induced by the field alone. This is manifested in the renormalization of the bands' chemical potentials $\mu_{1} \equiv \mu-\zeta_{1}$ and $\mu_{2} \equiv \mu+\zeta_{2}-E_{g}$, as well as the form of the distributions $f_{1}\left(\mu_{1}\right)$ and $f_{2}\left(\mu_{2}\right)$.

Extending the standard thermodynamic expression [17] for the Helmholtz free energy in each band, we write its difference with the energy of assembly as

$$
\begin{aligned}
F\left[f_{j}\left(\mu_{j}\right)\right] \equiv & 2 \int d k\left(k^{2}-\mu_{j}\right) f_{k j} \\
& +2 \int d k\left(f_{k j} \ln f_{k j}+\left(1-f_{k j}\right) \ln \left(1-f_{k j}\right)\right)
\end{aligned}
$$

recognizing the leading right-hand integral in equation (4) as the total internal energy, less the assembly energy. The second right-hand integral is the Uehling-Uhlenbeck entropy entering into the $\mathrm{H}$-theorem for fermions [18]. At global equilibrium, minimizing $F$ (understood as a functional of the distribution $f_{j}$ and subject to the latter's variation) leads to the familiar Fermi-Dirac form for $f_{j}$.

We recapitulate.

- A given gate voltage fixes the global chemical potential and total density within the channel.

- The source-drain field, acting independently of this, excites the carriers so a portion from the lower band is promoted to the upper band.

- The density decrease in the lower band is determined by the decrement $\zeta_{1}$ in the value of the common chemical potential $\mu$, while the increase in the upper band is determined by the increment $\zeta_{2}$ in $\mu$.

- Any loss from the lower band must match the gain in the upper one. Thus the two shifts in chemical potential are coupled by the conservation relation (3).

- To close the self-consistent solution for the $\zeta_{j}$, we look for any change in the free energy of the system as defined in equation (4), induced solely by application of the driving field. 
The behaviour of $F$ above, purely as a function of chemical potential, is quite different from its behaviour purely as a functional of $f$. In the equilibrium state it is readily seen that

$$
\frac{d F}{d \mu}[\bar{f}(\mu)]=-2 \int d k \bar{f}_{k}(\mu)
$$

so $F$ has no lower bound with increasing sub-band density. Since the form of the $f_{j}$ is strictly prescribed by solving the kinetic equations, the behaviour of $F$ is strictly a function of $\mu_{1}$ and $\mu_{2}$. The total net energy of the system, based on equation (4), must then exhibit maxima in the space $\left(\zeta_{1}, \zeta_{2}\right)$.

As our working principle we look for the maximum in the total net energy summed over both bands, as a function of the $\zeta_{j}$. The physical picture is analogous to a tap continuously feeding fluid to a finite container, which finally overflows. In a similar way the driven system will accumulate as much of the inflowing excess energy as it can, up to the point that increasing resistive dissipation matches the inflow and precludes any further internal buildup.

Next we construct the difference of the sum of net energies over the non-equilibrium distributions, indexed by $\zeta_{1}$ and $\zeta_{2}$, and its analogous non-equilibrium sum with $\zeta_{2}$ set to zero. This difference vanishes for $\zeta_{2}=0$. Otherwise, according to circumstances, it may exhibit a nontrivial maximum in parameter space as $\zeta_{2}$ is increased systematically. The computed quantity is

$$
\Delta F\left(\mu ; \zeta_{1}, \zeta_{2}\right)=\sum_{j=1,2}\left(F\left[f_{j}\left(\mu_{j}\right)\right]-\left.F\left[f_{j}\left(\mu_{j}\right)\right]\right|_{\zeta_{2}=0}\right) .
$$

Wherever we find the local maximum of $\Delta F$ on the locus of constant density $n(\mu)$ there is a unique self-consistent pair $\left(\zeta_{1}, \zeta_{2}\right)$. This fixes the desired physical solution for the interacting system. If no nontrivial solution exists, the maximum defaults to the $\zeta_{1}$-axis (that is, $\zeta_{2}=0$ ) and produces the standard Landauer conductance.

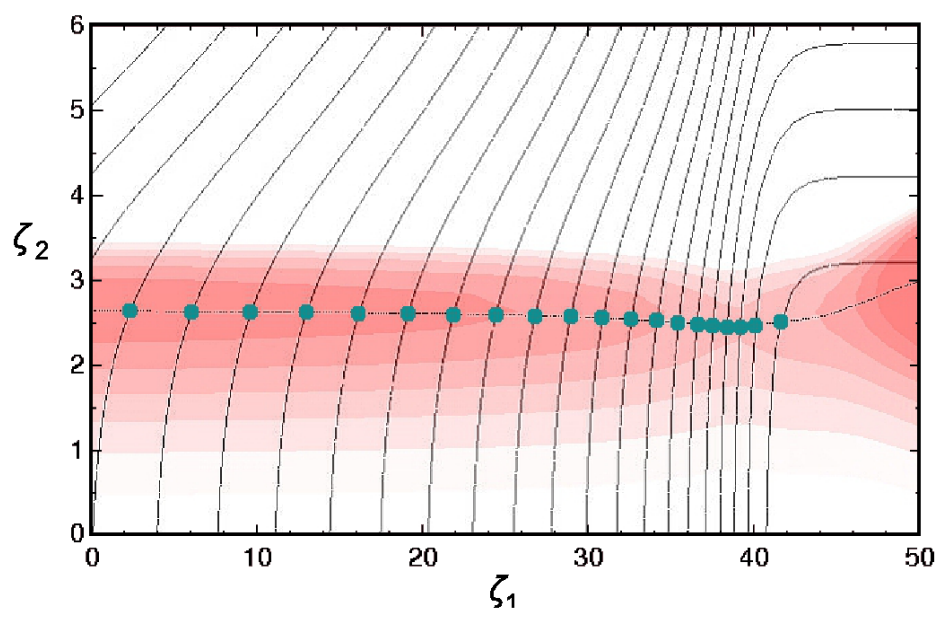

FIG. 2 Landscape of self-consistent solutions as functions of $\zeta_{1}$ and $\zeta_{2}$ (in thermal units) at $1 \mathrm{mV}$ source-drain voltage over a channel $2 \mu \mathrm{m}$ long, at temperature $4 \mathrm{~K}$. The dotted horizontal line at $\zeta_{2} \approx 2.6$, tracks the maximum in the bias-induced excess free energy $\Delta F\left(\mu ; \zeta_{1}, \zeta_{2}\right)$, equation (5), over the latter's density map. At global chemical potential $\mu$ the contours of constant channel density $n(\mu)$, equation (3), rise vertically from the $\zeta_{1}$-axis to intersect the maximum $\Delta F$ at the points of self-consistency (dots). For each fixed density the solution determines the system's non-linear conductance as a function of $\mu$. Since the physical chemical potential is $\mu=\mu_{0}-\zeta_{1}^{\prime}$ at the point of departure $\zeta_{1}^{\prime}$ on the $\zeta_{1}$-axis (with $\zeta_{1}^{\prime}=0$ at origin), the self-consistent values $\left(\zeta_{1}, \zeta_{2}\right)$ referred to $\mu_{0}$ are offset to yield the physical renormalizations $\left(\zeta_{1}-\zeta_{1}^{\prime}, \zeta_{2}+\zeta_{1}^{\prime}\right)$ referred to the physical $\mu$. 
Figure 2 illustrates the typical landscape of non-equilibrium net free energy and total electron density in the space of the renormalized chemical potentials, encompassing the family of selfconsistent solutions for a range of densities down from a given equilibrium starting value $n\left(\mu_{0}\right)$. The contour of constant density that matches the equilibrium value $n\left(\mu_{0}-\zeta_{1}^{\prime}\right)$, say, is superimposed on the contours of constant excess energy $\Delta F$. For $\zeta_{2}=0$ the upper band is practically empty so each contour of constant density departs orthogonally from the $\zeta_{1}$-axis. The landscape is mapped as $\zeta_{2}$ increases and the density in the upper band goes from near-empty to degenerate.

\subsection{Implementation}

Equation (5) is a measure of the non-equilibrium excess energy built up in the system when the driving field induces redistribution of the populations between sub-bands. Computing the self-consistent solution requires us to connect the intra-band MFPs, together of course with the inter-band transition probability, to the rates $R_{\mathrm{in} j}, R_{\mathrm{el} j}$ and $R_{0 j}$ that parametrize the collision terms in the coupled equations (1).

It is assumed that the mean free paths are common to each sub-band, though this need not be so more generally. Let $\lambda_{\text {in }}$ be the inelastic MFP and $\lambda_{\text {el }}$ be the elastic MFP. (Recall that, by hypothesis, the operational channel length is given by $L=\lambda_{\text {in }}$.) Any equilibrium distribution has its associated characteristic velocity

$$
\bar{v}(\mu) \equiv-\frac{v_{\text {th }}}{\bar{f}_{0}(\mu)} \int_{0}^{\infty} k d k \frac{\partial \bar{f}_{k}}{\partial k}(\mu)=v_{\text {th }}\left(1+e^{-\mu}\right) \int_{0}^{\infty} d k \bar{f}_{k}(\mu) .
$$

We have scaled out the thermal velocity $v_{\text {th }}=\hbar k_{\mathrm{th}} / m^{*}$ where $m^{*}$ is the electron effective mass. In the low-density classical limit, this becomes essentially $v_{\text {th }}$ while in the high-density degenerate limit it is the Fermi velocity $v_{\text {th }} \sqrt{\mu}$. The quantity $\bar{v}$ thus sets the typical velocity scale. Accordingly we define the rates in dimensionless units from the respective characteristic velocities:

$$
R_{\mathrm{in} j} \equiv \quad \frac{L}{v_{\mathrm{th}}} \frac{\bar{v}\left(\mu_{j}\right)}{\lambda_{\mathrm{in}}} ; \quad R_{\mathrm{el} j} \equiv \frac{L}{v_{\mathrm{th}}} \frac{\bar{v}\left(\mu_{j}\right)}{\lambda_{\mathrm{el}}}
$$

The transition rates represent a different physical mechanism and are treated differently from the intra-band ones, as a single dimensionless parameter

$$
R_{0 j} \equiv L / \lambda_{0}
$$

scaling inversely with a nominal "transition MFP" $\lambda_{0}$ which, however, is qualitatively distinct from intra-band MFPs. Its value is an experimental unknown. Moreover $\lambda_{0}$ is likely to depend strongly on device geometry and electrostatics [8]. For this work we set it an order of magnitude larger than the operational length $L$.

The current response summed over both parabolic sub-bands is given by

$$
\begin{aligned}
I\left(\mu, V_{\mathrm{sd}}\right) & \equiv q k_{\mathrm{th}} v_{\mathrm{th}} \int k \frac{d k}{\pi} \sum_{j=1,2} f_{k j}\left(\mu_{j}\right) \\
& =V_{\mathrm{sd}} \frac{q^{2}}{\pi \hbar} \int k d k \sum_{j=1,2}\left(\frac{\hbar k_{\mathrm{th}} v_{\mathrm{th}}}{q \mathcal{E} L} f_{k j}^{o}\left(\mu_{j}\right)\right)
\end{aligned}
$$

where the source-drain voltage is $V_{\text {sd }}=\mathcal{E} L$, and we note that the even distributions do not contribute. From equation (8) all the transport properties are derived. 


\section{Results}

We come now to the consequences for the quantized conductance. In figure 3 below, for a total channel current $I\left(\mu, V_{\mathrm{sd}}\right)$ at a series of fixed applied $V_{\mathrm{sd}}$, we show the computed chord conductance $G=I / V_{\text {sd }}$ for a device conforming to the specifications of reference [8]. The dynamical scattering parameters are those of the preceding section, namely: operational length $L=\lambda_{\text {in }}=2 \mu \mathrm{m} ; \lambda_{\mathrm{el}}=0.769 L=1.538 \mu \mathrm{m} ; \lambda_{0}=10 L=20 \mu \mathrm{m}$.

Figure 4 of reference [9] gives some evidence of thermal broadening of conductance at the sub-band thresholds presumably from localized Joule heating. We compute our curves at the nominal temperature $4 \mathrm{~K}$. The energy-gap value $E_{g}=15 \mathrm{meV}$ and the effective mass for GaAs are used. Finally, on the horizontal axis of our figure 3 we have mapped the global chemical potential $\mu$ to values of a corresponding gate-control voltage, using the parameters provided by reference 8 .

Our figure 3 should be compared directly with figure 1 as taken from reference [9], figure $3(\mathrm{a})$. Both in the real data of figure 1 and in our calculation, the action of a substantial sourcedrain voltage driving the current through the channel leads to a series of elevated conductance plateaux which

(a) are inherently non-linear in origin,

(b) are extremely flat and robust,

(c) anomalously exceed the Landauer upper bound on $G$ and thus

(d) violate the unitary limit of linear-response transmission theory.

The striking confluence of behaviours between experiment and theory speaks for itself.

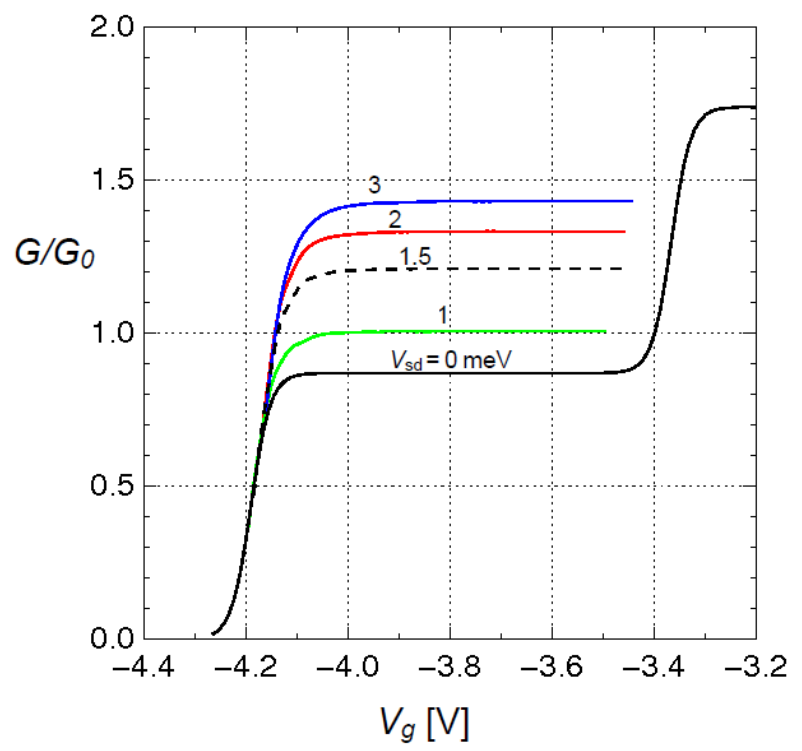

FIG. 3 Anomalous enhancement of conductance $G$ calculated for a ballistic device equivalent to that of figure 1 (figure $3(\mathrm{a})$ of reference [9) at a nominal temperature $4 \mathrm{~K}$. Axis scales are as for that figure; $G$ is plotted (units of $G_{0}=q^{2} / \pi \hbar$ ) versus gate voltage $V_{g}$ sweeping the channel density across the energy gap from the bottom of the ground-state sub-band to the threshold of the first excited-state sub-band. Bottom curve: in weak-field response the quantized conductance matches that for standard linear response. Higher curves: as the driving voltage $V_{\mathrm{sd}}$ increases, the conductance acquires a non-linear enhancement. The action of inter-band transitions dynamically redistributes carrier density between subbands. This is responsible for the strong enhancement of the step in $G$, beyond the unitarity limit posited by quantum-transmission theories of conductance. 
In figure 3(a) of reference [9] (and figure 1 reproducing it in this paper) the plots show $\partial I / \partial V_{\text {sd }}$ : the rate of change of current with driving field, plotted as the density increases. It is easily seen that when that slope is essentially flat over a broad range of gate voltage (thus density) as in figure 1 , the simple conductance $I / V_{\mathrm{sd}}$, as in figure 3 , must track it closely and vice versa.

Before examining further characteristics of our theoretical conductance we discuss differences between the present implementation and the experiment. Our calculation here exhibits greater sensitivity with respect to $V_{\text {sd }}$ than the experiment so that, while in figure 3 the step increase of $G$ resulting from $V_{\mathrm{sd}}=1 \mathrm{mV}$ coincides with that in figure 1 , its height at $3 \mathrm{mV}$ is $1.43 G_{0}$ while its counterpart in figure 1 is $1.16 G_{0}$. This overestimate might be accounted for in part if the inelastic MFP $\lambda_{\text {in }}$ suffered shortening via optical-phonon emission at higher driving voltages (the optical-phonon energy in GaAs is $35 \mathrm{meV}$, not hugely larger than $E_{g}$ at $15 \mathrm{meV}$ ). Use of energydependent mean free paths within equations (1), rather than fixed ones, is an obvious aspect for exploration. Increased local Joule heating with increased driving field may also suppress the plateaux, as figure 4 suggests.

In figure 4 we show the properties of the enhanced conductance taken at a typical midgap density where $G$ is steady. Device specifications are the same as for figure 3, with an additional choice of temperature, $8 \mathrm{~K}$ as well as $4 \mathrm{~K}$. Our calculated $G$ exhibits an onset at finite field and asymptotic saturation at high fields. The threshold voltage value at onset depends on temperature; at low temperature the threshold value of $V_{\text {sd }}$ tends to zero and at high temperatures it rises in rough proportion to $T$.

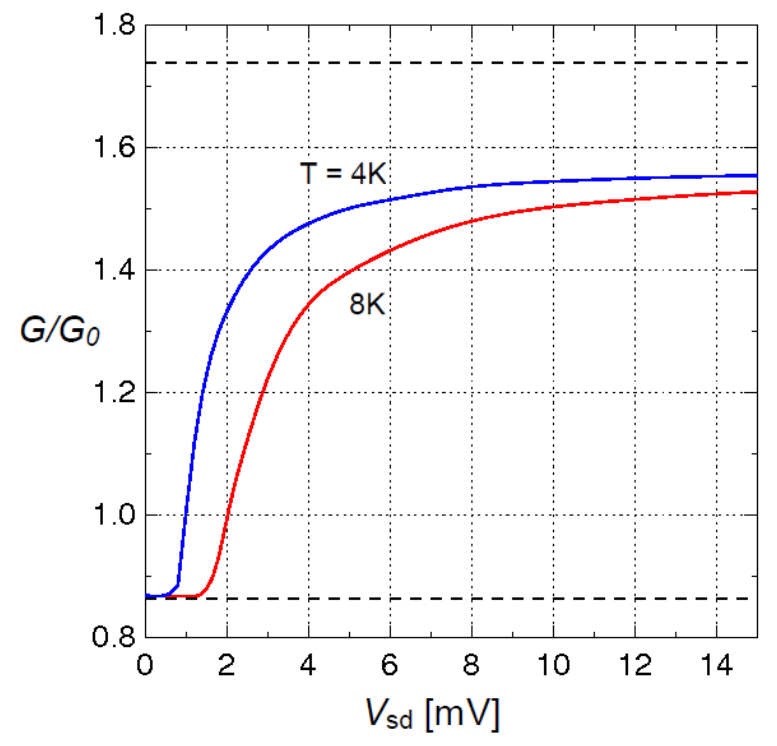

FIG.4 Threshold and saturation behaviour of calculated conductance versus source-drain voltage, at temperatures $T=4 \mathrm{~K}$ and $8 \mathrm{~K}$. Lower dotted line is the first Landauer level in this model, upper line is the second level. The threshold driving field for onset of the enhancement scales approximately with $T$. The saturation asymptote at high fields is independent of temperature.

The phenomenon above may partly explain why measurements prior to de Picciotto et al [8, 9] have not recorded the anomalous enhancement. Predominantly, experiments in quantized conductance have been carried out either at weak fields below threshold, or at higher temperatures, or on noisy devices, or in any combination of the above. Any enhancement of $G$ under such conditions would tend to be washed out.

Saturation of $G$ in figure 4 sets in at driving fields considerably higher than those employed 
in reference [8] and in our figure 3. The upper bound of $G$ is close to $1.6 G_{0}$, well short of the second occupied level at $1.77 G_{0}$ in the weak-field limit. This suggests that there is a field- and temperature-independent maximum transfer of carriers to the upper band, beyond which the feedback of transitions returning carriers to the lower band precludes any increase. Saturation behaviour may provide a further experimental window on the dynamics of the interband transition.

\section{Summary and Implications}

De Picciotto et al [8, 9] in the first instance addressed their experiments to the topic (still unresolved) of the " 0.7 anomaly". Yet the same data harbours a message that is perhaps more seminal to the understanding of ballistic transport at low temperatures; namely, the quite surprising violation of the unitary limit for quantized conductance.

That violation is illusory; the apparent paradox vanishes when a more appropriate kinetictheoretical argument is brought to bear, going beyond the limits of single-particle transmission theory. Carrier transitions between well separated sub-bands are generation-recombination processes, viewed microscopically. This means that their quantitative description must address the direct creation and destruction of actual occupancies in such discretely separate energy bands. Unitarity still applies but, playing out as it must on the much larger stage of multiparticle dynamics, it cannot be accommodated by purely single-particle prescriptions.

Put succinctly, inelasticity and thermodynamic irreversibility rule the physics. It is crucial to build these into the theory explicitly. That is not feasible within the restrictive confines of reversible single-particle Hamiltonian dynamics.

De Picciotto and co-authors have presaged a role for inter-band transitions in the dynamics of their structures [8]. Our motive here has been to advance a theory of such transitions in terms of textbook quantum kinetics.

Certainly the boundary conditions for this problem do require special care in interpretation to be given to the ballistic nature of mesoscopic one-dimensional conductors. Nevertheless it is unavoidable to confront a transport problem where both elastic and inelastic scattering processes act with equal physical status, as in all normal metallic transport.

Our calculation strongly reinforces the conjecture [8] that inter-band transitions in a 1D ballistic device do indeed produce anomalous enhancement of the quantized conductance, within the density regime between a sub-band threshold and its next-higher neighbour's. This enhancement can indeed readily exceed the presumed unitarity limit set by $G_{0}$, mandated as the absolute stepwise upper bound for 1D conductance.

The straightforward reason for that phenomenon rests with the physics of creationannihilation across a band gap and is not beholden to single-particle conservation band-by-band, as it were in isolation. Only global conservation, subsuming the bands within one interacting system, applies. The central mechanism is non-linear feedback between the coupled sub-band populations.

Finally, beyond explaining theoretically the quizzical enhancement of ballistic conductance reported in references [8] and [9], our results on temperature behaviour from figure 4 offer a basis to predict thermal characteristics for transition-induced changes in conductance in clean quantum wires. Furthermore, there is a case for probing similar effects in sufficiently clean carbon nanotubes, whose energy scale and robustness at high fields far outstrip any device based on GaAs heterojunction technology. All of this would call for novel experiments.

\section{References}

[1] Ferry D K and Goodnick S M 2009 Transport in Nanostructures 2nd ed (Cambridge: Cambridge University Press) 
[2] Davies J 1998 Physics of Low-Dimensional Semiconductors: An Introduction (Cambridge: Cambridge University Press)

[3] Nazarov Y V and Blanter Y M 2009 Quantum Transport: Introduction to Nanoscience (Cambridge: Cambridge University Press)

[4] Landauer R D 1996 J. Math. Phys 37

[5] Imry Y and Landauer R 1999 Rev. Mod. Phys. 71 S306

[6] Neder I, Heiblum M, Levinson Y, Mahalu D and Umansky V 2006 Phys. Rev. Lett. 96016804

[7] Green F, Thakur J and Das M P 2004 Phys. Rev. Lett. 92156804

[8] de Picciotto R, Pfeiffer L N, Baldwin K W and West K W 2004 Phys. Rev. Lett. 92, 036805

[9] de Picciotto R, Baldwin K W, Pfeiffer L N and West K W 2008 J. Phys.: Condens. Matter 20164204

[10] Magnus W and Schoenmaker W 2002 Quantum Transport in Submicron Devices (Berlin: Springer)

[11] Das M P and Green F 2012 J. Phys.: Condens. Matter 24183201

[12] Das M P and Green F 2003 J. Phys.: Condens. Matter 15 L687; 2009 J. Phys.: Condens. Matter 21101001

[13] Green F and Das M P 2005 Fluctuation and Noise Letters 5 C1

[14] Das M P and Green F 2017 Adv. Nat. Sci.: Nanosci. Nanotechnol. 8023001

[15] Greene M P, Lee H J, Quinn J J and Rodriguez S 1969 Phys. Rev. 1771019

[16] Mermin N D 1970 Phys. Rev. B 12363

[17] Kittel C 1958 Elementary Statistical Physics (New York: Wiley)

[18] Uehling E A and Uhlenbeck G E 1933 Phys. Rev. 43552 